\section{Summary points}

The PROGRESS trial reports that a "perindopril-based

blood-pressure-lowering regimen" provided significant protection against recurrent stroke

Only the subgroup receiving both perindopril and indapamide had reduced stroke recurrence; the study design did not include a subgroup randomised to indapamide alone (previously shown to reduce stroke recurrence)

Some reviews of the PROGRESS trial have advocated using perindopril alone for prevention of stroke recurrence, which does not follow from the findings

Results from major drug trials must be clearly presented to avoid misinterpretation by busy clinicians

Contributors and sources: The authors are both academic physicians with an interest in language and how it is used in scientific discourse. RW is a neurologist working mainly in the fields of epilepsy and electroencephalography; CZ is an internist working mainly in the fields of palliative care and social science in medicine. This article was written after the PROGRESS trial was brought to the authors' attention by events surrounding the management of a patient in their teaching hospital.

Competing interests: None declared.

1 PROGRESS Collaborative Group. Randomised trial of a perindoprilbased blood-pressure-lowering regimen among 6,105 individuals with a previous stroke or transient ischaemic attack. Lancet 2001;358:1033-41.

2 Staessen JA, Wang J. Blood-pressure lowering for the secondary prevention of stroke [comment]. Lancet 2001;358:1026-7.
3 Cates C. The lowering of blood pressure after stroke [letter]. Lancet 2001;358:1993.

4 Tirschwell D. Combined therapy with indapamide and perindopril but not perindopril alone reduced the risk for recurrent stroke. ACP J Club 2002;136:51.

5 Psaty BM, Weiss NS, Furberg CD. The PROGRESS trial: questions about the effectiveness of angiotensin converting enzyme inhibitors. $\mathrm{Am}$ Hypertens 2002;15:472-4.

6 Psaty BM, Smith NS, Siscovick DS, Koepsell TD, Weiss NS, Heckbert SR, et al. Health outcomes associated with antihypertensive therapies used as first-line agents: a systematic review and meta-analysis. JAMA 1997;277:739-45.

7 PATS Collaborating Group. Post-stroke antihypertensive treatment study. A preliminary result. Chin Med J 1995;108:710-7.

8 Neal B, MacMahon S, Chapman N. Blood Pressure Lowering Treatment Trialists' Collaboration. Effects of ACE inhibitors, calcium antagonists and other blood pressure lowering drugs: results of prospectively designed overviews of randomised trials. Lancet 2000;355:1955-64.

9 Staessen JA, WangJG, Thijs L. Cardiovascular protection and blood pressure reduction: a meta-analysis. Lancet 2001;358:1305-15.

10 Turnbull F. Blood Pressure Lowering Treatment Trialists' Collaboration. Effects of different blood-pressure lowering regimens on major cardiovascular events: results of prospectively-designed overviews of rancardiovascular events: results of prospectiv
domised trials. Lancet 2003;362:1527-35.

11 PROGRESS Collaborative Group. Effects of a perindopril-based blood pressure lowering regimen on cardiac outcomes among patients with cerebrovascular disease. Eur Heart J 2003;24:475-84.

12 PROGRESS Collaborative Group. Effects of blood pressure lowering with perindopril and indapamide therapy on dementia and cognitive decline in patients with cerebrovascular disease. Arch Intern Med 2003:163:1069-75.

13 Fransen M, Anderson C, Chalmers J, Chapman N, Davis S, MacMahon S, et al. Effects of a perindopril-based blood pressure-lowering regimen on disability and dependency in 6105 patients with cerebrovascular disease: disability and dependency in 6105 patients with cereb
a randomized controlled trial. Stroke 2003;34:2333-8.

14 Chapman N, Huxley R, Anderson C, Bousser MG, Chalmers J, Colman S, et al. Effects of a perindopril-based blood pressure-lowering regimen on the risk of recurrent stroke according to stroke subtype and medical history: the PROGRESS trial. Stroke 2004;35:116-21.

15 Tonkin AM. Does lowering blood pressure prevent recurrent stroke? Med J Aust 2002;176:283-4.

16 Britov AN, Bystrova MM, Orlov AA. Control of arterial hypertension in stroke prevention. Klin Med (Mosk) 2002;80:53-7.

17 Jackson G. Making PROGRESS in stable patients post stroke or transient ischemic attack: implications for general practice. Int J Clin Pract 2003;57:385-7.

\title{
Commentary: The PROGRESS trial three years later: time for more action, less distraction
}

\author{
Stephen MacMahon, Bruce Neal, Anthony Rodgers, John Chalmers
}

The George

Institute for

International

Health, University

of Sydney, PO Box

M201, Sydney, NSW

2050, Australia

Stephen

MacMahon

professor of

cardiovascula

medicine and

epidemiology

Bruce Neal

associate professor of

medicine

John Chalmers

emeritus professor of

medicine

Clinical Trials

Research Unit,

University of

Auckland,

Auckland, New

Zealand

Anthony Rodgers

director

Correspondence to:

S MacMahon

smacmahon@

thegeorgeinstitute.org
Since the publication of the results of the PROGRESS trial, there has been much comment in the BMJ and elsewhere. ${ }^{12}$ Most of this acknowledges the importance of the findings for the care of patients with cerebrovascular disease. These patients are at high risk of stroke recurrence, and before the trial was completed few interventions had been proved to reduce this risk. Aspirin was known to modestly reduce the risk of recurrence of ischaemic stroke, but no treatment had been shown to reduce the frequently catastrophic recurrence of cerebral haemorrhage. This situation was changed profoundly by the results of PROGRESS, which showed that a simple blood pressure lowering regimen substantially reduced the risks of recurrent stroke, ${ }^{3}$ disability, ${ }^{4}$ and cardiac events ${ }^{5}$ across a broad range of blood pressure levels in patients with either ischaemic or haemorrhagic cerebrovascular disease.

PROGRESS was conceived during an era in which many stroke specialists were concerned about possible risks of blood pressure lowering in patients with compromised cerebral circulation. While epidemiological evidence indicated that the lowest blood pressure levels were associated with the lowest risks of stroke recurrence, ${ }^{6}$ prevailing clinical opinion required us to allow individual doctors the discretion to determine the intensity of the blood pressure lowering regimen they provided to individual patients. The treatment regimen in the study comprised the angiotensin converting enzyme inhibitor perindopril and the diuretic indapamide. Our objective was to have as many patients as possible treated with both drugs; however, the treating doctors decided whether to introduce indapamide after patients had been stablised on perindopril.

Although PROGRESS was not designed to determine the separate effects of perindopril and indapamide, the benefits of combination treatment with these agents proved to be particularly large, with stroke risks cut by almost a half and reductions in most other cardiovascular outcomes, including death. Conversely, treatment with perindopril alone provided no detectable benefits, although the $95 \%$ confidence intervals were wide and modest benefits could not be excluded. Not surprisingly, therefore, the principal conclusion was that patients with cerebrovascular disease should be considered routinely for combination treatment with perindopril and indapamide. ${ }^{3}$ This regimen 
would prevent at least one major event among every 10 patients treated for five years, ${ }^{5}$ an outcome that would materially improve prognosis.

The importance of these findings was clear to those responsible for developing major new guidelines for the treatment of hypertension ${ }^{78}$ and stroke. ${ }^{9}$ However, the same cannot be said for a few commentators, ${ }^{10}$ who have sought to focus debate on the comparative effects of the two drugs used in the study, even though PROGRESS provides no useful information about this. A pervasive fixation with the comparative effects of different drugs has unduly influenced recent debate about the effects of blood pressure lowering. Much of this has been fuelled by pharmaceutical companies claiming supposedly unique effects of their own drugs or adverse effects of competitors drugs, but there have also been counterclaims by special interest groups of unique protective effects of off-patent drugs and adverse effects of on-patent drugs. However, in large scale randomised trials comparing different treatment regimens, the evidence of "special" drug effects has mostly proved evanescent. ${ }^{11}$

What is clearer is that the size of the blood pressure reduction is a major determinant of outcome, ${ }^{11}$ a finding consistent with the observation in PROGRESS that combination therapy produced larger reductions in blood pressure and stroke risk than did a single drug. Some have argued that the observed difference in outcomes cannot be explained by differences in blood pressure,,${ }^{10}$ but this is refuted by data on the predicted effects of blood pressure reduction among patients with cerebrovascular disease. ${ }^{6}$ The predicted and observed effects of the overall blood pressure reduction achieved in PROGRESS $(9 / 4 \mathrm{~mm} \mathrm{Hg})$ are identical (figure). Additionally, the observed effects of combination therapy and single drug therapy are not significantly different from those predicted, although the confidence limits for the estimates of observed effects were wide.

The finding that combination therapy produces benefits at least as large as those predicted by epidemiological studies provides strong support for the recommendation that stroke patients should be considered routinely for treatment with both perindopril and indapamide. ${ }^{3}$ Although PROGRESS provides no evidence about the effects of other blood pressure lowering drugs, there is no particular reason to believe that other regimens would not confer benefits, and in resource poor settings where access to perindopril and indapamide is limited it would seem reasonable to recommend alternate agents. However, continuing the academic debate about the effects of different blood pressure lowering agents for the secondary prevention of stroke only retards the implementation of the findings of PROGRESS. If patients are denied intensive blood pressure lowering treatment as a consequence, unnecessary suffering will result. For the sake of the 50 million people worldwide with cerebrovascular disease, let's set aside arguments about elusive special drug effects and focus on the issue of prime importance. To adapt the well known words of a recent US president: "It's the blood pressure, stupid."

Contributors: $\mathrm{SM}, \mathrm{BN}, \mathrm{AR}$ and JC all contributed to initial and subsequent drafts of this commentary. Arier Lee conducted the

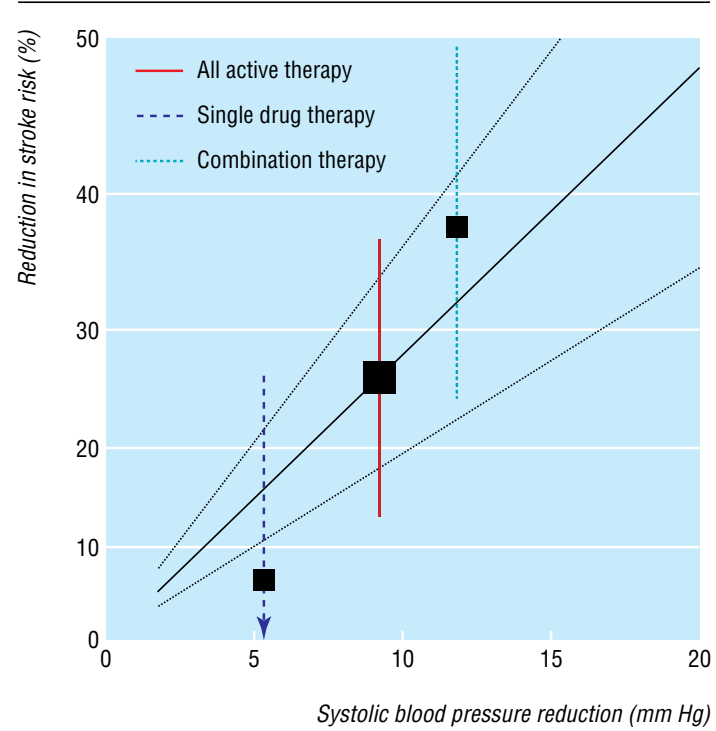

Predicted and observed effects of blood pressure lowering among patients with ischaemic stroke or transient ischaemic attacks. Predicted reductions in stroke risk and 95\% confidence interval for a given reduction in usual systolic blood pressure are indicated by diagonal lines ${ }^{6}$; Observed effects of treatment on stroke risk and $95 \%$ confidence intervals are indicated by vertical lines and boxes. ${ }^{3}$ Box sizes are proportional to the number of strokes. All estimates of reductions in stroke risk are plotted on a logarithmic scale

statistical analyses comparing predicted and observed effects of blood pressure reduction in PROGRESS; Jeffery Cutler, Charles Warlow, and Mark Woodward advised on the interpretation of those analyses.

Competing interests: $\mathrm{BN}$ is the recipient of a career development award from the Heart Foundation of Australia. AR is a senior fellow of the National Heart Foundation of New Zealand. PROGESS was supported by grants from the Health Research Council of New Zealand, the National Health and Medical Research Council of Australia and the Institut de Recherches Internationale Servier. PROGRESS was designed, conducted, analysed, and reported by the collaborating investigators independently of all sponsors. Each of the authors has received research grants and travel expenses or lecture honorariums from one or more of these three study sponsors.

1 Wennberg R, Zimmermann C. The PROGRESS trial three years later: time for a balanced report of effectiveness. BMJ 2004;329:968-70.

2 Muir KW. Secondary prevention for stroke and transient ischaemic attacks. BMJ 2004; 328:297-8.

3 PROGRESS Collaborative Group. Randomised trial of a perindoprilbased blood-pressure-lowering regimen among 6105 individuals with previous stroke or transient ischaemic attack. Lancet 2001;358:1033-41.

4 PROGRESS Collaborative Group. Effects of a perindopril-based blood pressure lowering regimen on disability and dependency in 6105 pressure lowering regimen on disability and dependency
patients with cerebrovascular diseases. Stroke 2003;34:2333-8.

5 PROGRESS Collaborative Group. Effects of a perindopril-based blood pressure lowering regimen on cardiac outcomes among patients with cerebrovascular disease. Eur Heart J 2002;24:475-84.

6 Rodgers A, MacMahon S, Gamble G, Slattery J, Sandercock P, Warlow C, et al. Blood pressure and risk of stroke in patients with cerebrovascular disease. BMJ 1996;313:147.

7 Seventh report of the Joint National Committee on Prevention, Detection, Evaluation and Treatment of High Blood Pressure: the JNC 7 report. JAMA 2003;289:2560-72.

8 Guidelines Committee. 2003 European Society of HypertensionEuropean Society of Cardiology guidelines for the management of arterial hypertension. J Hypertens 2003;21:1011-53.

9 Intercollegiate Working Party for Stroke. National clinical guidelines for stroke. Update 2002. London: Royal College of Physicians, 2002.

10 Psaty BM, Weiss NS, Furberg CD. The PROGRESS trial: questions about the effectiveness of angiotensin-concerting enzyme inhibitors. Am J Hypertens 2002; 15:472-4.

11 Blood Pressure Lowering Treatment Trialists' Collaboration. Effects of different blood-pressure-lowering regimens on major cardiovascular events: results of prospectively-designed overviews of randomised trials. Lancet 2003;362:1527-35. 